\title{
Development trend of world men decathlon scores BP neural network analysis
}

\author{
Chao Chen ${ }^{1, a}$ and Bing Zhang ${ }^{2, b}$ \\ ${ }^{1}$ Institute of Physical Education, Jilin Normal University, Siping 136000, China \\ ${ }^{2}$ Institute of Physical Education, Huanggang Normal University, Huangzhou 438000, China
}

\begin{abstract}
Using BP neural network prediction model is established, with the methods of principal component analysis was carried out on the statistical data, obtained the four principal components. Based on the results of principal component analysis, the BP neural network model is established. With principal component analysis (pca) for the input, with the result of data processing to solve more than men's decathlon single movement data and the correlation of each single performance is stronger for shall not influence brought by the prediction accuracy of forecasting model, to improve the prediction precision of the prediction model, extending the application of the BP neural network prediction model.
\end{abstract}

\section{Introduction}

Sports' performance prediction accuracy extent directly affects athletes training plan making and training schedule, and has bigger impacts on athlete performance enhancement. By far, in the aspect of applying BP neural network prediction model to predict sports performance, domestic and foreign lots of scholars have made relative studies. BP neural network can make full use of existing information, and predicts future sports performance by previous sports performances. Principal component analysis method can utilize thought of dimension reduction to reduce indicators numbers, and solve inconvenience that is brought to model establishment by indicators amount and correlations in problems to be solved. Principal component analysis approach and BP neural network model have been widely used in relative sports field problems, and the combination of the two will also provide new ideas to solve sports problems [1-3].

The paper makes improvement on BP neural network prediction model, applies principal component analysis method to handle with statistical data, reduces BP neural network input dimensions, and finally on the basis of BP neural network prediction model, it establishes men decathlon performance prediction model, promotes men decathlon performance prediction accuracy while at the same time analyzes and researches on principal component method and BP neural network prediction model combinative model's application in sports prediction $[4,5]$.

\section{Data statistics and preprocessing}

a,b Corresponding author: ${ }^{\text {a } 398704001 @ q q . c o m ; ~}$ btiyuxi@qq.com

\subsection{Men decathlon annual highest performance data statistics}

In order to promote prediction accuracy on world men decathlon annual highest performance, its statistical data not only is required to make statistics of previous world men decathlon annual highest performances athletes total performances, but also is required to make statistics of their each individual event performance so as to make more effective mining on their total performances change rules.

The paper makes statistics of world men decathlon annual highest performances in ten years during 2004 2013, and based on this, it establishes prediction model. Year 2004 2013 world men decathlon annual highest performances statistical data is as Table 1 shows.

\subsection{Statistical data processing}

The paper's prediction on world men decathlon event annual highest performance is prediction on their total performances, in data prediction, prediction methods that often use are traditional regression prediction, time sequence prediction and else, while also recent years newly-developed grey prediction, fuzzy prediction and neural network predication and so on. For traditional prediction model and grey prediction model and else, their data changes should follow certain change rules, and each prediction model's prediction on different change rules data also have their own advantages and disadvantages.

In order to easier analyzing world men decathlon annual highest performances change trend during 
2004 2013 so as to easy selecting different prediction models, the paper processes with Table 1 total performances data, in Table 1 data total performances changing curve is as Figure 1 shows.

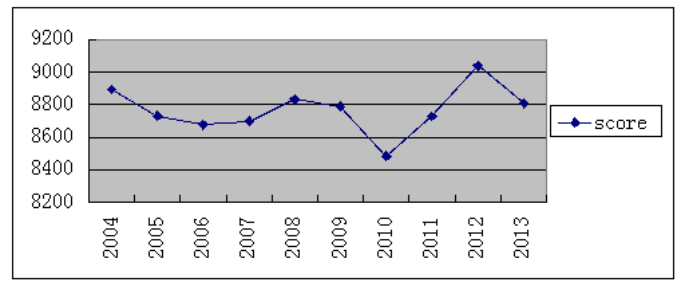

Figure1. 2004 2013 world men decathlon annual highest performances changing graph..

By Figure 1, it is clear during ten years from 2004 to 2013, men decathlon world annual highest performances are in fluctuation changes, from which performances in 2010 and 2012 are respectively the lowest performance and highest performance in the ten years. Due to performance changing trend and changing rules are not obvious, traditional statistics methods are hard to establish prediction model on them, so the paper selects BP neural network model to establish prediction model on them [6].
Due to Table 1 statistical data dimensions are different, each individual event performance and total performance statistical data values changes are bigger, and it is bad for its principal component analysis and BP neural network prediction model establishment. For the convenience of the paper's men decathlon performance prediction model establishment, the paper carries on normalization processing on them, for total performance, long jump, shot put, high jump, discus, pole vault and javelin throw these seven events performances, statistical data gets bigger, it shows performances are better, and other four individual events performances are just the opposite. So for total performance and long jump so on seven events, take 2004 total performance and each individual event performance as denominator, and take 2004 2013 corresponding total performance and each individual event performance as numerator, carry on processing. For $100 \mathrm{~m}, 400 \mathrm{~m}, 110 \mathrm{~m}$ hurdle and $1500 \mathrm{~m}$ these four individual events, it takes 2004 total performance and each individual event performance as numerator, and takes 2004 2013 corresponding total performance and each individual event performance as denominator, carries on processing. Their processing result is as Table 2 shows.

Table 1. World men decathlon annual highest performance.

\begin{tabular}{ccccccccccc}
\hline Year & $\mathbf{2 0 0 4}$ & $\mathbf{2 0 0 5}$ & $\mathbf{2 0 0 6}$ & $\mathbf{2 0 0 7}$ & $\mathbf{2 0 0 8}$ & $\mathbf{2 0 0 9}$ & $\mathbf{2 0 1 0}$ & $\mathbf{2 0 1 1}$ & $\mathbf{2 0 1 2}$ & $\mathbf{2 0 1 3}$ \\
\hline $\begin{array}{c}\text { Total } \\
\text { performance }\end{array}$ & 8893 & 8732 & 8677 & 8697 & 8832 & 8790 & 8483 & 8729 & 9039 & 8809 \\
$\mathbf{1 0 0 m}$ & 10.85 & 10.43 & 10.42 & 10.94 & 10.39 & 10.45 & 10.35 & 10.33 & 10.21 & 10.35 \\
Long jump & 7.84 & 7.54 & 7.67 & 7.84 & 7.39 & 7.83 & 7.51 & 7.8 & 8.23 & 7.73 \\
Shot put & 16.36 & 16.25 & 15.56 & 16.47 & 15.17 & 15.33 & 15.38 & 14.14 & 14.20 & 14.39 \\
High jump & 2.12 & 2.00 & 2.06 & 2.12 & 2.08 & 1.99 & 2.06 & 2.05 & 2.05 & 1.93 \\
400m & 48.36 & 47.78 & 48.87 & 48.99 & 48.41 & 48.13 & 49.66 & 46.35 & 46.70 & 46.02 \\
110m hurdle & 14.05 & 14.43 & 13.74 & 14.39 & 13.75 & 13.86 & 14.08 & 13.52 & 13.70 & 13.72 \\
Discus & 48.72 & 53.68 & 52.21 & 47.66 & 52.74 & 48.08 & 49.85 & 41.58 & 42.81 & 45.00 \\
Pole vault & 5.00 & 4.90 & 5.00 & 4.80 & 5.00 & 5.20 & 4.60 & 5.05 & 5.30 & 5.20 \\
Javelin throw & 70.52 & 72.00 & 66.47 & 68.87 & 70.55 & 68.00 & 66.1 & 56.19 & 58.87 & 64.83 \\
1500m running & 300.0 & 303.8 & 313.5 & 280.4 & 290.9 & 288.0 & 296.4 & 264.1 & 254.5 & 269.8 \\
\hline
\end{tabular}

Table 2. World men decathlon annual highest performance preprocessing result.

\begin{tabular}{cccccccccccc}
\hline Year & $\mathbf{2 0 0 4}$ & $\mathbf{2 0 0 5}$ & $\mathbf{2 0 0 6}$ & $\mathbf{2 0 0 7}$ & $\mathbf{2 0 0 8}$ & $\mathbf{2 0 0 9}$ & $\mathbf{2 0 1 0}$ & $\mathbf{2 0 1 1}$ & $\mathbf{2 0 1 2}$ & $\mathbf{2 0 1 3}$ \\
\hline $\begin{array}{c}\text { Total } \\
\text { performance }\end{array}$ & 1.000 & 0.982 & 0.976 & 0.978 & 0.993 & 0.988 & 0.954 & 0.982 & 1.016 & 0.991 \\
100m & 1.000 & 1.040 & 1.041 & 0.992 & 1.044 & 1.038 & 1.048 & 1.050 & 1.063 & 1.048 \\
Long jump & 1.000 & 0.962 & 0.978 & 1.000 & 0.943 & 0.999 & 0.958 & 0.995 & 1.050 & 0.986 \\
Shot put & 1.000 & 0.993 & 0.951 & 1.007 & 0.927 & 0.937 & 0.940 & 0.864 & 0.868 & 0.880 \\
High jump & 1.000 & 0.943 & 0.972 & 1.000 & 0.981 & 0.939 & 0.972 & 0.967 & 0.967 & 0.910 \\
$\quad$ 400m & 1.000 & 1.012 & 0.990 & 0.987 & 0.999 & 1.005 & 0.974 & 1.043 & 1.036 & 1.051 \\
110m hurdle & 1.000 & 0.974 & 1.023 & 0.976 & 1.022 & 1.013 & 0.998 & 1.039 & 1.026 & 1.024 \\
Discus & 1.000 & 1.102 & 1.072 & 0.978 & 1.083 & 0.987 & 1.023 & 0.853 & 0.879 & 0.924 \\
Pole vault & 1.000 & 0.980 & 1.000 & 0.960 & 1.000 & 1.040 & 0.920 & 1.010 & 1.060 & 1.040 \\
Javelin throw & 1.000 & 1.021 & 0.943 & 0.977 & 1.001 & 0.964 & 0.937 & 0.797 & 0.835 & 0.919 \\
1500m & 1.000 & 0.988 & 0.957 & 1.070 & 1.031 & 1.042 & 1.012 & 1.136 & 1.179 & 1.112 \\
\hline
\end{tabular}


Table 3. Solved four principal components values.

\begin{tabular}{ccccccccccc}
\hline Year & $\mathbf{2 0 0 4}$ & $\mathbf{2 0 0 5}$ & $\mathbf{2 0 0 6}$ & $\mathbf{2 0 0 7}$ & $\mathbf{2 0 0 8}$ & $\mathbf{2 0 0 9}$ & $\mathbf{2 0 1 0}$ & $\mathbf{2 0 1 1}$ & $\mathbf{2 0 1 2}$ & $\mathbf{2 0 1 3}$ \\
\hline $\begin{array}{c}\text { First principal } \\
\text { component }\end{array}$ & 1.376 & 1.272 & 1.408 & 1.404 & 1.418 & 1.607 & 1.412 & 2.037 & 2.077 & 1.872 \\
$\begin{array}{c}\text { Second } \\
\text { principal } \\
\text { component }\end{array}$ & 0.300 & 0.163 & 0.186 & 0.352 & 0.170 & 0.241 & 0.216 & 0.340 & 0.368 & 0.245 \\
$\begin{array}{c}\text { Third principal } \\
\text { component } \\
\text { The fourth }\end{array}$ & 0.727 & 0.753 & 0.685 & 0.704 & 0.693 & 0.735 & 0.635 & 0.615 & 0.669 & 0.720 \\
principal \\
component
\end{tabular}

\section{Prediction system establishment}

BP neural network prediction model is a kind of simple and steepest descent optimizing method, it can fully excavate data relationship, it has good effects on sports performance prediction problems with fewer data information amount, but BP prediction system itself has certain drawbacks, as when input space is seriously self-correlated and network dimensions are higher, its prediction effects will go bad [5]. In order to promote men decathlon performances prediction accuracy, the paper needs to apply all effective data that are sports competitions' total performances and individual events performances. Due to ten individual events partial events correlations are higher, it surely causes established BP neural network model input data dimensions are higher and correlations are big, it affects model prediction accuracy. The paper applies principal component analysis to handle with data, and on the basis of handled data, it establishes BP neural network prediction model.

The paper established BP neural network prediction model has input layer, output layer and hidden layer, from which hidden layer has one layer nerve cell, model selects $\mathrm{S}$ type tangent function transfer function and pure linear function, gradient descent momentum BP gain function, gradient descent momentum weight learning function and mean square error normalization property function. Among them, parameter $a_{\text {and }} \eta$ are respectively 0.5 and 0.2 , neural network maximum training times are 10000 times, training accuracy is 0.005 , training indication interval is 1000 times. Output layer nerve cell number is 1 , input layer nerve cell number is number of principal components after principal component analysis handling, hidden layer nerve cells amount is solved by formula $i=\sqrt{n+m}+a$, from which $a$ value is between 0 and 1 .

\section{Model result and result analysis}

By above analysis, it is clear that in the first principal component, the larger loading values components are shot put, discus, javelin throw and $1500 \mathrm{~m}$ four events. Among them, shot put, discus and javelin throw all belong to throwing type event, $1500 \mathrm{~m}$ belongs to endurance type event, which shows the two types of events have largest impacts on world men decathlon annual highest performance. In the second principal components, larger loading components are $100 \mathrm{~m}$, long jump and high jump three events, the three is related to athletes lower limbs strength and explosive power. In the third principal component, larger loading components are high jump and pole vault. In the fourth principal component, larger loading events are $400 \mathrm{~m}$ and $110 \mathrm{~m}$ hurdle. By above analysis, it is clear that solved four principal components contain world men decathlon annual highest performances' each event information to very high extent. Established principal component analysis model analysis effects on men decathlon world annual best performance is good, it can precede with BP neural network prediction model analysis [7].

By above solved loading amount, it can solve 2004 2013 four principal components values as Table 3 shows.

The paper established neural network prediction model uses previous year four principal components values to predict next year total performance. By above analysis, it is clear, established BP neural network prediction model system input has four principal components values that input layer has four nerve cells, set neural network output and four principal components corresponding next year men decathlon total performance value that output layer nerve cell unit number is one. According to input layer and output layer nerve cell number, it defines hidden layer nerve cell number is three. The paper takes year 2004 2011 data as training mode, uses year 2012 and 2013 two years data to detect established model, and apply year 2013 data to predict year 2014 annual highest performance. Then training mode input matrix P1 and output matrix $\mathrm{T} 1$ are respectively as following:

$$
\begin{aligned}
& P 1= {\left[\begin{array}{lllllll}
1.376 & 1.272 & 1.408 & 1.404 & 1.418 & 1.607 & 1.412 \\
0.300 & 0.163 & 0.186 & 0.352 & 0.170 & 0.241 & 0.216 \\
0.727 & 0.753 & 0.685 & 0.704 & 0.693 & 0.725 & 0.635 \\
1.221 & 1.202 & 1.237 & 1.270 & 1.224 & 1.207 & 1.165
\end{array}\right] } \\
& T 1=\left[\begin{array}{lllllll}
0.982 & 0.976 & 0.978 & 0.993 & 0.988 & 0.954 & 0.982
\end{array}\right]
\end{aligned}
$$

Utilize set training mode to train BP neural network model, its training process curve graph is as Figure 2 shows. 


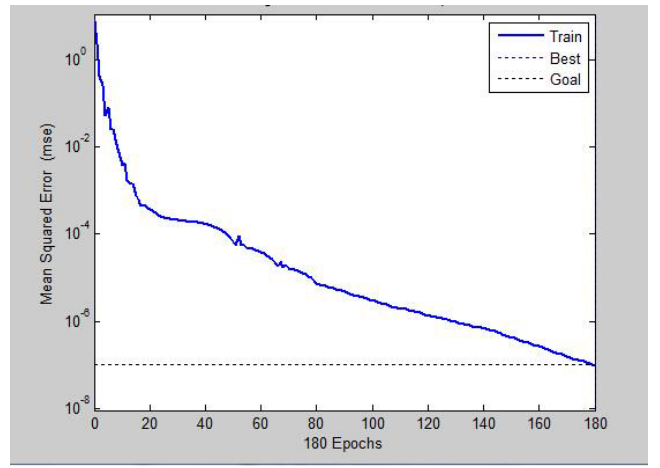

Figure 2. Training process curve.

As Figure 2, neural network model through 180 times training, its model accuracy then arrives at set accuracy, its convergent effects are also good. Respectively input year 2011 and year 2012 data into established BP neural network prediction model, its prediction results are respectively 1.0063 and 1.0000 , errors with the two years corresponding actual values 1.0164 and 0.9906 are respectively $0.99 \%$ and $0.95 \%$, prediction accuracy is very high. Input year 2013 data into prediction model, it gets its predicted value is 0.9840 , multiply it by year 2004 highest performance, then it get year 2014 annual highest performance prediction model is 8750.7 .

\section{Conclusions}

Men decathlon world annual highest prediction has important significances in world men decathlon development, but due to its performance change trend fluctuation is stronger, change rules are not obvious, traditional prediction model is hard to make prediction model on it. The paper applies BP neural network prediction model to model it, but men decathlon each event performance correlation is bigger and individual event data is numerous and other problems surely will bring bad impacts to BP neural network modeling. To solve above problems bad impacts on modeling, the paper applies principal component analysis to process with statistical data; take established principal components as input, and total performance as output. It solves above problem, and meanwhile, it promotes established prediction model's prediction accuracy.

\section{References}

1. Z.H. Zhao, Journal of Shenyang Sport University 3 (2003).

2. X.M. Chen, Bulletin of Sport Science \& Technology 15 (2007).

3. Z. Lan, Sport Science And Technology 29 (2008).

4. D.P. Zhong, X.Y. Zhang, Journal of Wuhan Institute of Physical Education 36 (2002).

5. Q. Hu, Journal of Physical Education Institute of Shanxi Teachers University 19 (2004).

6. Z.L. Liu, S. Masuko, K. Solakyildirim, Biochemistry, 49, 45 (2010).

7. N.R. Leite, A. Magalhaes, L.M. Silva, Journal of Coastal Research (2016). 